\title{
Indicadores de idoneidad didáctica en matematica: propuesta para una experiencia de "aprendo en casa", programa de educación virtual en Perú
}

Indicators of didactic suitability in mathematics: proposal for an "I learn at home" experience, virtual education program in Peru

Indicadores de aptidão didática em matemática: proposta de experiência "Eu aprendo em casa", programa de educação virtual no Peru

Franklin Taipe Florez

ftaipef@unsa.edu.pe

Universidad Nacional de San Agustin de Arequipa - Peru https://orcid.org/0000-0002-3639-3892

Mario Sacsi Quispe

sacsiquispemario@gmail.com Universidad Andina Nestor Caceres Velasquez de Juliaca - Peru https://orcid.org/0000-0001-6298-7945

Dino Aymachoque Alvarado dinoaymachoque@gmail.com Universidad Nacional del Altiplano Puno - Peru https://orcid.org/0000-0003-1389-6486

Neptali Condori Cutipa jahirco1601@gmail.com Universidad Nacional Jorge Basadre Grohmann de Tacna - Peru https://orcid.org/0000-0002-5310-3404

Belinda Caceres Mendigure b16linda@gmail.com Universidad Nacional San Antonio Abad del Cusco - Peru https://orcid.org/0000-0001-8571-048X

\section{RESUMEN}

Existen estudios sobre "aprendo en casa" que es la plataforma multicanal para la educación virtual del ministerio de educación en Perú referente a su alcance, logística y problemáticas, no a su idoneidad didáctica, el objetivo del estudio es proponer indicadores contextualizados de idoneidad didactica para las dimensiones "epistémica" de situacionesproblema, lenguaje y "ecológica" de adaptación al currículo y sociocultural especialmente, conforme al enfoque ontosemiotico de la instrucción matematica, se utiliza el enfoque cualitativo interpretativo y de alcance exploratorio, tipo no experimental transeccional con la técnica del focus group, donde un grupo colegiado de siete docentes conocedores del enfoque ontosemiotico analizaron y desarrollaron la experiencia de aprendizaje "Preferencias de productos para un negocio" de la evaluación diagnostica para el quinto grado de primaria, en base a la competencia resuelve problemas de gestión de datos e incertidumbre. El colegiado docente propuso tres indicadores básicos genéricos indispensables con los cuales los estudiantes pueden evidenciar el logro de la competencia matemática desarrollada para cada componente de acuerdo a las capacidades de representar información con graficos, comunicar las ideas estadísticas, usar estrategias de solución y argumentar soluciones con información procesada, adecuados al contexto de la educación virtual en zonas rurales, en concordancia al estándar de ciclo y componentes de idoneidad didactica, los resultados sugieren con urgencia la necesidad del trabajo colegiado, colaborativo permanente para generar instrumentos de análisis y evaluación didáctica, indicadores adecuados y contextualizados a la realidad de la educación virtual rural.

Palabras clave / Descriptores: idoneidad didáctica, gestión de datos, indicadores, ontosemiótico, educación virtual.

\section{ABSTRACT}

There are studies on "I learn at home" which is the multichannel platform for virtual education of the Ministry of Education in Peru regarding its scope, logistics and problems, not its didactic suitability, the objective of the study is to propose contextualized indicators of didactic suitability for the "epistemic" dimensions of problem-situations, language 
and "ecological" of adaptation to the curriculum and sociocultural, especially, according to the ontosemiotic approach of mathematical instruction, the qualitative interpretative approach and exploratory scope are used, a non-experimental transectional type with the technique of the focus group, where a collegiate group of seven teachers familiar with the ontosemiotic approach analyzed and developed the learning experience "Preferences of products for a business" of the diagnostic evaluation for the fifth grade of primary school, based on the competence solves management problems data and uncertainty. The teaching staff proposed three essential basic indicators with which students can demonstrate the achievement of the mathematical competence developed for each component according to the abilities to represent information with graphics, communicate statistical ideas, use solution strategies and argue solutions with information. process, appropriate to the context of virtual education in rural areas, in accordance with the cycle standard and components of didactic suitability, the results urgently suggest the need for collegial, permanent collaborative work to generate instruments for didactic analysis and evaluation, adequate indicators and contextualized to the reality of rural virtual education

Keywords: didactic suitability, data management, indicators, ontosemiotic, virtual education.

\section{RESUMO}

Existem estudos sobre "Eu aprendo em casa" que é a plataforma multicanal para educação virtual do Ministério da Educação do Peru no que se refere a sua abrangência, logística e problemas, não sua adequação didática, o objetivo do estudo é propor indicadores contextualizados de didática adequação às dimensões "epistêmica" de situações-problema, linguagem e "ecológica" de adaptação ao currículo e sociocultural, especialmente, de acordo com a abordagem ontosemiótica do ensino de matemática, utiliza-se a abordagem qualitativa interpretativa e de âmbito exploratório, de caráter não experimental tipo transversal com a técnica do grupo focal, onde um grupo colegiado de sete professores familiarizados com a abordagem ontosemiótica analisou e desenvolveu a experiência de aprendizagem "Preferências de produto para uma empresa" da avaliação diagnóstica para o quinto ano do ensino fundamental, com base no competência resolve dados de problemas de gestão e incerteza. A equipa pedagógica propôs três indicadores básicos genéricos essenciais com os quais os alunos podem demonstrar o alcance da competência matemática desenvolvida para cada componente de acordo com as capacidades de representar informação com gráficos, comunicar ideias estatísticas, utilizar estratégias de solução e argumentar soluções com informação processada, adequada para No contexto da educação virtual no meio rural, de acordo com os componentes padrão do ciclo e adequação didática, os resultados sugerem urgentemente a necessidade de um trabalho colegiado e colaborativo permanente para gerar instrumentos didáticos de análise e avaliação, indicadores adequados e contextualizados à realidade do rural virtual. Educação.

Palavras-chave / Descritores: adequação didática, gerenciamento de dados, indicadores, ontossemiótica, educação virtual.

\section{INTRODUCCIÓN}

En el contexto de la emergencia sanitaria por el Covid-19, el ministerio de educación en el Perú, ofrece la estrategia de educación virtual multicanal "aprendo en casa" por radio, TV e Internet a todos los estudiantes en los diferentes niveles y modalidades del país, este servicio tiene serias dificultades por los problemas de brecha digital, cobertura logística, contenidos, adecuaciones y otros (Hernandez, 2020; Zavala \& Franco, 2020), pero a la vez constituye un hito pedagógico para el sistema educativo peruano por la masificación de las TICs ya que la estrategia atiende de manera universal a todos los niveles y modalidades de la educación peruana (Jacobo, 2020). Los estudios sobre la influencia del Covid-19 en educación se centran en los de tipo influencia en la salud, acceso a la educación virtual, recursos tecnológicos uso software y aplicativos entre otros (Hernández, 2020) que a su vez brindan oportunidades para generar respuestas urgentes e innovadoras, existen estudios sobre generación de espacios afectivos para el aprendizaje (CaceresPiñazola, 2020), pero son raros los estudios sobre aspectos como la calidad de contenido, los instrumentos, recursos, materias impartidas en la educación virtual.

La estrategia ofrece experiencias de aprendizaje, para el trabajo de múltiples áreas curriculares que buscan el logro de capacidades y competencias (CNEB, 2016) similares al trabajo por enfoque basado en proyectos, para el área de matemática en el nivel primario, se pretende el logro de competencias una de ellas es resuelve problemas de gestión de datos e incertidumbre, del área de matematica para que los estudiantes analicen datos, tomen decisiones, planteen 
predicciones, y conclusiones con respaldo en la información producida con el trabajo de las capacidades de: representa datos con gráficos y medidas estadísticas e probabilísticas, comunica la comprensión de los conceptos estadísticos y probabilísticos, usa estrategias y procedimientos para recopilar y procesar datos, sustenta conclusiones o decisiones con base en información obtenida, que tiene analogía con los principios de la (NTCM, 2000) en sus principios para la acción para asegurar el logro matemático de todos, como lo refiere tambien (Scott, 2018). Que enseñar y cómo hacerlo es un problema pedagógico y de la educación matemática, cuando se asume como ciencia del diseño (Lesh y Sriramn, 2010). Dicho problema incumbe a los responsables de los diseños curriculares y ejecutores del diseño (profesores) con quienes se pone en operacion directa, por lo cual resulta de importancia adaptar al contexto instrumentos existentes en la teoría de la didáctica matemática (Scott, 2018).

Al iniciar el año escolar 2021, se implementa la evaluación diagnostica como parte de la evaluación para ver el estado del desarrollo de las competencias de los estudiantes, como indica (Ravela, 2017) que un acto educacional es un proceso gris que requiere ser visibilizado a través de un proceso sistemático de evaluación. Estas evaluaciones proporcionan información sobre el nivel de logro de las competencias del o de la estudiante, y a partir de ella se pueden plantear, diseños de contención, oportunidades de mejora, conforme la disposición gubernamental (RVM Nº 093-2020MINEDU), cumpliendo una función pedagógica importante para el proceso de enseñanzaaprendizaje (Vera, 2020; Saavedra, 2019). El instrumento para la evaluación diagnostica nacional 2021 que corresponde al nivel de educación primaria en el portal "aprendo en casa", para la experiencia de aprendizaje con la situación significativa "Preferencias de productos para un negocio" (https://repositorio.perueduca.pe/docentes/orientaciones/planificacion/primaria/fasciculomatematica.pdf), tomándose como referencia los estándares de estas competencias para el ciclo quinto de la Educación Básica Regular, resuelve problemas de cantidad y de gestión de datos e incertidumbre (CNEB, 2016). Este y cualquier otro instrumento de planificación y diseño tiene que estar sujeto a evaluación de su idoneidad didáctica para procurar mejoras continuas desde el diseño como lo indica (Godino, 2002).

El trabajo colegiado docente por la emergencia sanitaria se realiza de manera virtual, este trabajo consiste en reuniones de estudio, debate, análisis y propuestas para el desarrollo de los procesos de enseñanza-aprendizaje para el logro de competencias (Diaz y Santos, 2020), es un nuevo modo, que al momento tiene ventajas y desventajas, pueden aprovecharse sus espacios para en análisis y propuesta de instrumentos de trabajo, que unidos a otras técnicas como el focus group podrían tener significancia en la producción de instrumentos, conocimientos, materiales, propuestasw, proyectos, y otros como lo señala Meo y Dabenigno (2020), que inciden en el problema de la brecha digital y la responsabilidad del docente en este contexto. La importancia que los maestros produzcan material virtual o desde la virtualidad, materiales para uso en su propio trabajo es significante aun en este tiempo de angustia, esta característica de la docencia es paret de su propia resiliencia y compromiso con su trabajo a entender de (Sanchez, Guzman y Delgado, 2021) que consideran al trabajo colegiado en virtualidad como capital professional entre la investigación educativa y su práctica pedagógica.

En muchos instrumentos de planficacion, evaluación para los estudiantes de manera individual o estandarizada no se tiene indicadores de idioneidad didáctica contextualizada al entorno de la educación rural virtual en época de Covid-19, esto constituye el problema al cual nos enfrentamos al momento de proponer estrategias de mejora o de metaevaluacion que mejoren los procesos (Diaz y Valdez, 2020). El objetivo del estudio es presentar una propuesta de indicadores de idoneidad didáctica conforme al enfoque ontosemiotico en sus dimensiones epistémica y ecologica, para la situación significativa "preferencias de productos para un negocio", como experiencia de aprendizaje en la competencia resuelve problemas de gestión de datos e incertidumbre, de la evaluación diagnostica con el programa "aprendo en casa".

Es justificable la realización de la investigación por que se requiere de instrumentos contextualizados a cada medio geográfico y condiciones de toda indole de los estudiantes para 
obtener resultados objetivos y que los mismo instrumentos puedan mejorarse continuamente como un ejercicio profesional docente y disciplina institucional de los agentes educativos. (Osorio, 2017) refiere en su estudio sobre análisis de los indicadores de idoneidad didáctica de los maestros de matemática que sus conocimientos son limitados a un análisis complejo y constructivos, por ello su necesidad e importancia., estos indicadores de idoneidad didáctica guarda relación con que "las competencias son procesos complejos de desempeño con idoneidad en un determinado contexto", como lo sugiere (Tobon, 2013). La idoneidad didáctica tiene varias aristas que justifican su uso y constante evaluación para mejorar el aprendizaje, siendo un componente el uso como una herramienta de teoría metodológica del enfoque ontosemiotico en el conocimiento de la instrucción matemática (Malet, Giacomone y Repetto, 2021).

La previsión sobre el planteamiento de indicadores de idoneidad didáctica para la prueba diagnostica en la experiencia de aprendizaje es que los docentes dispongan de una construcción para los indicadores en analogía a las herramientas metodológicas de las cuales hacen uso en la planificación y evaluación del diseño de sus propios instrumentos de clase.

Para el fundamento teorico se utilizo el enfoque de la teoría ontosemiótica del conocimiento y la instrucción matemática (EOS, enfoque ontosemiotico) que propusieron los investigadores (Godino, Batanero y Font, 2007), el enfoque ontosemiotico adquiere importancia por asociar el conocimiento matemático con su practica para cubrir las propiedas necesidades del aprendiz y que se puedan mejorar continuamente los procesos de planificación y enseñanza desde el cuerpo docente o profesorado. En el aspecto de la metodología se aprovecho el colegiado virtual para utilizar la técnica del focus group y tener un análisis constructivo del instrumento para plantear indicadores de idoneidad didáctica como un ejercicio profesional docente constante. Los resultados expresan los indicadores minimos que debe tener una evidencia como resultado de la evaluación diagnostica con referencia a las capacidades de la competencia, el estándar de ciclo y los componentes de las dimensiones en la idoneidad didáctica. La sección de resultados presenta además de los indicadores la discusión de estos con referentes bibliográficos y en las conclusiones se refiere alcances para implementar los indicadores de idioneidad didáctica asi como para el trabajo colegiado en el análisis de instrumentos.

\section{FUNDAMENTO TEÓRICO}

El diseño educativo en la didáctica del "enfoque ontosemiótico" del conocimiento y la instrucción matemática (EOS) (Godino, Batanero y Font, 2007), es el marco teórico que, como describe D“eAmore y Godino (2007), es el desarrollo de la teoría antropológica en didáctica de la matemática (Chevallard, 1992; 1999), el enfoque EOS, y su noción de idoneidad didáctica, puede aportar elementos originales y significativos para elaborar una teoría de diseño instruccional, apropiada para orientar los procesos de enseñanza y aprendizaje de las matemáticas y otras áreas curriculares (Godino, 2011). Una teoría de la instrucción en un área de contenido específico no puede dar recetas de actuación para cada circunstancia, pero sí principios y criterios generales (Godino, 2010) por ello es preciso generar indicadores para los componentes de las dimensiones de idoneidad didáctica, según el contexto de aplicación.

Los términos de idoneidad didáctica, sus dimensiones, componentes y criterios, se introdujeron en el EOS (Godino, Contreras y Font, 2006; Godino, Bencomo, Font y Wilhelmi, 2007) como herramienta que permite el paso de una didáctica descriptiva - explicativa a una didáctica normativa, ósea una didáctica de intervención efectiva en el aula (Godino, 2011). Actualmente se usa el marco de referencia del Enfoque Ontosemiótico (EOS), (Godino, Batanero y Font, 2007) en investigaciones en el ámbito de la enseñanza de la estadística, entre ellas, la variedad que usan la herramienta de la idoneidad didáctica para mostrar su aplicabilidad tanto en evaluar implementaciones de aula como en formaciones de profesorado de matemáticas (Breda, Pino-Fan y 
Font, 2017; Font, Planas y Godino, 2010; Nogueira, Fernández y Rodríguez Vivero, 2015; Ramos, Fagúndez y Castells, 2009).

El Enfoque Ontosemiótico (EOS) plantea la idoneidad didáctica como el instrumento sistémico de un proceso de instrucción, sesión de aprendizaje, proyecto educativo, experiencia de aprendizaje, módulo de aprendizaje (Berciano, et.al., 2020), cuyo principal indicador empírico es la adaptación entre significados personales logrados y significados institucionales pretendidos o implementados (Godino, Wilhelmi y Bencomo, 2005; Godino, Contreras y Font, 2006). Para el éxito de una idoneidad didáctica satisfactoria de un proceso de instrucción se requiere tener en cuenta de manera sistémica seis dimensiones (Godino, Batanero y Font, 2007):

1) Idoneidad epistémica: correspondencia entre los contenidos o significados implementados y los de referencia.

2) Idoneidad ecológica: adecuación del proceso de enseñanza y aprendizaje al entorno social $\mathrm{y}$ proyecto de centro.

3) Idoneidad cognitiva: adecuación de los significados implementados a los conocimientos previos y diversidad de características del alumnado.

4) Idoneidad afectiva: implicación del alumnado en el proceso implementado.

5) Idoneidad interaccional: potencialidad del proceso enseñanza-aprendizaje para identificar y resolver posibles conflictos semióticos.

6) Idoneidad mediacional: disponibilidad y adecuación de medios para el desarrollo del proceso de instrucción.

Cada dimensión está formada por componentes (Tabla 1); y para cada componente se propone un conjunto de indicadores de idoneidad con el fin de garantizar su consecución (Godino, 2013).

Tabla 1.

Componentes asociados a cada dimensión de la idoneidad didáctica.

\begin{tabular}{ll}
\hline Dimension & \multicolumn{1}{c}{ Componente } \\
\hline Epistémica & Situaciones problema, lenguajes, reglas, argumentos, relaciones \\
Ecológica & $\begin{array}{l}\text { Innovación, adaptación sociocultural, curricular y profesional, conexiones intra e } \\
\text { interdisciplinares. }\end{array}$ \\
Cognitiva & $\begin{array}{l}\text { Conocimientos previos, adaptacion curricular a diferencias individuales, aprendizajes. } \\
\text { Afectiva }\end{array}$ \\
Interaccional actitudes, emociones. \\
Mediacional & $\begin{array}{l}\text { Interacción docente-dicente, interacción entre discentes, autonomía. } \\
\text { Recursos materiales, número de estudiantes, condiciones de aula, tiempo para la } \\
\end{array}$
\end{tabular}

Fuente: (Berciano, et. al, 2020)

La descripción de idoneidad didáctica de una práctica docente matemática supone un análisis multidimensional complejo (Berciano, et.al, 2020). Vemos ejemplos en investigaciones acerca de la enseñanza y aprendizaje de la estadística y probabilidad que indican la necesidad de mejorar la formación en la componente mediacional del futuro profesorado de estadística (Arteaga, Batanero y Gea, 2017), o la importancia del conocimiento de los indicadores asociados a la idoneidad afectiva en la enseñanza de la probabilidad (Beltrán-Pellicer y Godino, 2017). Pero son raros los estudios que analizan las repercusiones de la educación estadística con la competencia "resuelve problemas en gestión de datos e incertidumbre" en el contexto de la educación virtual 
con escasa logística de conectividad y en el aislamiento de los estudiantes en educación primaria, concretamente en la educación virtual rural en época de pandemia del Covid-19.

En el estudio de Cruz et al.(2017), sobre los criterios de idoneidad epistémica en geometría espacial en niños del nivel primario se propone un sistema de criterios específicos de idoneidad para la faceta epistémica que son aplicados sobre resultados de indagaciones de los estudiantes, estos criterios no pueden considerarse como universales y absolutos sino como un instrumento para apoyar el análisis de la idoneidad didáctica, como el argumento de Godino, Batanero, Font y Giacomone (2016) este ejercicio debe ser una competencia mas del desarrollo profesional docente para su uso pertinente, continuo y valorativo.

La investigación de (Arjona, 2020) referente al análisis de la idoneidad didáctica en videos educativos de Youtube con el tema de funciones en segundo grado ESO, en la que concluye que existe una diferencia muy fuerte entre una visión teorica de una clase ideal desde el enfoque ontosemiotico de la idoneidad didáctica y las llevadas a practica por fierentes fuentes como el Youtube y otros medios virtuales, incidiendo que la teoría de la idoneidad didactiva no se plasma en los videos observándose una brecha que debe ser reducida con el alcance pedagógico de la teoría de la instrucción matemática.

Con respecto a la técnica del focus groups, es una herramienta en la investigación cualitativa, su ventaja radica en obtener los reales intereses y necesidades de los participantes, además de guiarse del rigor de las etapas de investigación, desde la planeación de la situación problemática, hasta alcanzar el informe de investigación (Ivancovich yAraya, 2011). En el caso de Yepes et al. (2017) utilizaron esta técnica como instrumento para obtener y processar información cualitativa, com el fin de indagar las necesidades, sensaciones, conjeturas y concepciones de los sujetos en investigacion. Tambien se a usado la técnica em estúdios pedagógicos como el reportado por Gomez et al. (2014) em su estúdio sobre equipos docentes em el nível de educacion superior como uma utopia o realidade, investigando-se por diagnostico la realidade de los equipos docentes siendo el focus group la técnica para reccolectar informacion. Asi como tambien reportan su uso em investigaciones sociales educativos en jovenes como menciona (Gomez-Escalonilla et al., 2015). Asi como tambien (Juan y Roussos, 2010) donde señalan que la tecnica del focus groups para el trabajo com relaciones humanas y com docentes em equipo tiene excelentes oportunidades de acceso y logro segun sus objetivos quedando evidenciado que es uma técnica de investigacion cualitativa valida.

\section{PROCEDIMIENTOS METODOLOGICOS}

La metodología del estudio fue cualitativa de diseño no experimental, transeccional (Hernandez-Sampieri, et. al, 2014), tiene el enfoque de la investigación interpretativa de tipo exploratorio, aplicándose el método de las investigaciones de diseño (Kelly, et. al, 2008) en una situación contextual virtual de análisis y propuesta para el diseño/planificación, y posterior implementación de indicadores de idoneidad didáctica en la experiencia de aprendizaje para la evaluación diagnostica del V ciclo de educación primaria con la estrategia de "aprendo en casa" en el arco de la educación virtual en el Peru, con la técnica del "focus group" donde participaron siete docentes con conocimientos teóricos y prácticos sobre la idoneidad didáctica desde el enfoque ontosemiotico (EOS), siendo esta una técnica de estudio de opiniones o actitudes que se realiza con pequeños grupos de personas, para indagar sobre aspectos específicos como indica (Cortes, 2008).

El estudio se realizó en el ámbito de la Ugel Canas, donde las condiciones de conectividad y logística para la implementación de la estrategia "aprendo en casa" son limitadas, el nivel de pobreza es generalizado y no se tiene un apoyo efectivo de los padres de familia hacia sus hijos, como un típico sector marginal que con la crisis sanitaria a reflejado sus verdaderas carencias que requieren de una atención urgente (Castañeda et al., 2020) la implementación de la investigación se realizó en un entorno estrictamente virtual y de trabajo remoto, las actividades fueron planeadas con 
reuniones de preparación, ejecución y evaluación en la plataforma Google meet, realizándose las siguientes actividades.

\subsection{Socializacion del enfoque}

Como primera actividad se realizo una socialización entre los investigadores y docentes expertos (del focus group), del enfoque ontosemiotico de la instrucción matemática (EOS), con ejemplificaciones en otras investigaciones y reportes de indicadores de investigadores como (Berciano, et. al, 2020; Godino, 2012; Breda, et. al. 2020, Breda, et. al, 2018; Breda, et. al. 2016; Giacomone, et. al, 2019; Godino, 2011; Ramos-Font, 2008), analizando principalmente contextos para indicadores de las dimensiones epistemica y ecológica de la idoneidad didáctica (EOS), teniendo como materiales de trabajo los componentes propuestos por Godino (2011) que para la dimensión epistémica, incluye a las situaciones-problemas; elementos lingüísticos representativos; elementos regulativos (conceptos/definiciones, procedimientos y proposiciones); argumentos y las relaciones entre dichos elementos como naturaleza de la actividad matemática, en concordancia con (Rivas, 2014). En el caso de la dimensión ecológica entendida como relación del plan para aprender matemática y su adecuación al entorno del contexto que se utiliza (Godino, 2011), asi la consideración de situaciones externas a tomar en cuenta para la instrucción matemática expuestas por (Rivas, 2014), esta dimensión tiene los componentes de: adaptación al currículo; apertura hacia la innovación didáctica; adaptación socio profesional y cultural; educación en valores y conexiones intra e interdisciplinarias.

Esta primera actividad se ejecuto en un tiempo sincronico de 03 horas y un tiempo asincrónico de 48 horas a libertad de los docnetes integrantes del colegiado en el focus group.

\subsection{Socializacion de la experiencia de aprendizaje}

En la segunda reunión se socializo la situación significativa de la experiencia de aprendizaje "Preferencias de productos para un negocio" para la evaluación diagnostica nacional 2021 del Ministerio de Educacion del Peru a estudiantes del V ciclo de educación primaria, en el marco de la estrategia de educación virtual "aprendo en casa", la solución de esta situación significativa por los estudiantes está diseñada en el marco del desarrollo de competencias (CNEB, 2016) en este caso las competencias resuelve problemas de cantidad y resuelve problemas de gestión de datos e incertidumbre, esta última fue analizad a profundidad para proponer los indicadores de idoneidad didáctica en el contexto de la educación rural, a distancia. El grupo de investigadores y los expertos del focus group, analizaron el estándar del ciclo para la competencia, las evidencias que deben producir los estudiantes (elaborar una encuesta u otro instrumento para el recojo de la información, representar en tablas de doble entrada o gráficos estadísticos de la información que se recoge, y lo registra en una hoja de trabajo, elaboración de cálculos, justifica el proceso de resolución, expresa su proceso y resultado haciendo uso del cálculo), las capacidades de la competencia resuelve problemas de gestión de datos e incertidumbre que son, representa datos con gráficos y medidas estadísticas o probabilísticas, comunica la comprensión de los conceptos estadísticos y probabilísticos, usa estrategias y procedimientos para recopilar y procesar datos, sustenta conclusiones o decisiones con base en información obtenida. (CNEB, 2016). Teniendo como material, la situación significativa de la evaluación diagnostica, figura 1, la cual es:

“Arturo, un niño del 4to grado, cuenta que cerca de la casa de su abuelo hay un establo, que vende rica leche y quesos. A él le encanta el queso, y dice que le gustaría ser dueño del establo para elaborar muchos quesos y disfrutar de este alimento, aunque sería mejor, tener una empresa de los mejores quesos del país".

Es posible que por el lugar donde vives hayas visitado un establo, o una tienda de productos lácteos, ¿qué otros productos venden allí?, o quizá cerca de tu casa o del colegio, tengas alguna tienda, o algún negocio de una familia, en las que venden productos de primera necesidad, entre 
menestras, detergente u otros. Piensa en las tiendas o negocios cerca de tu colegio, ¿qué productos venden? ¿qué productos consideras que son los preferidos de tus amigos y los tuyos? En esta actividad, deberás considerar que, muchos niños acuden con algún familiar a las tiendas para comprar productos de su agrado, por lo tanto, deberás pensar en desarrollar algunas actividades que te permitan dar recomendaciones a los dueños de las tiendas, sobre los productos que deberán vender si quisieran tener más ventas y más clientes. ¿Cuánto dinero podrán obtener con la compra de los productos preferidos? Para recoger información sobre los gustos, y preferencias en la compra de productos, de tus amigos y familiares, es posible que elabores una propuesta de estos productos en base a los cuales ellos podrán elegir. Observa, en las tiendas cercanas a tu casa o a tu escuela, ¿qué productos venden?, ¿qué utilidad tienen?, ¿son productos de tu preferencia?

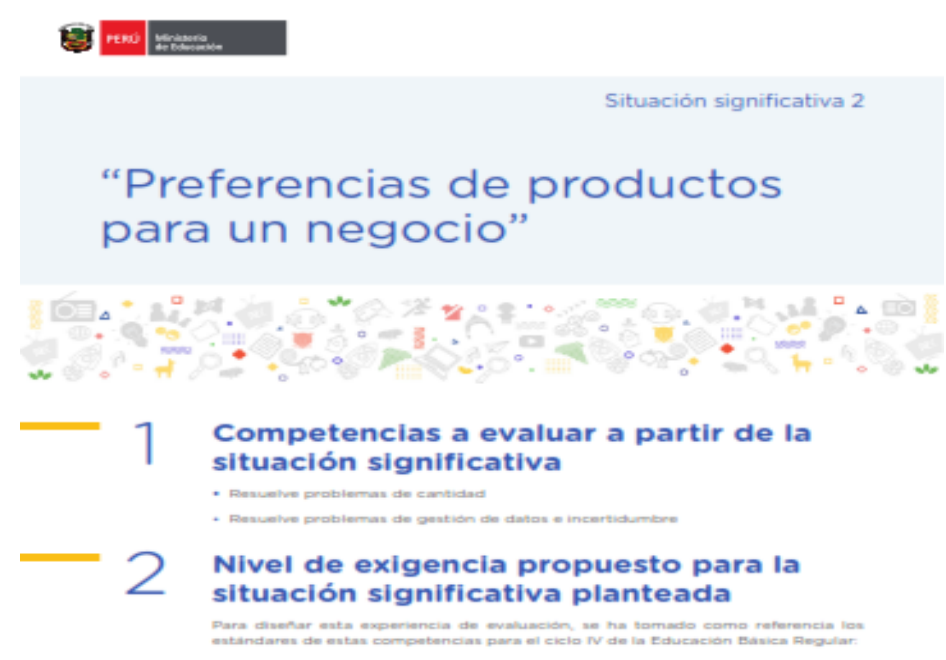

Figura 1: Situación significativa para la evaluación diagnostica disponible en: https://repositorio.perueduca.pe/docentes/orientaciones/planificacion/primaria/fasciculomatematica.pdf.

\subsection{Analisis y resolución individual}

Esta fue realizada de manera asincrónica, cada experto durante tres días, analizo individualmente la situación significativa, el contexto, los posibles indicadores, el estándar, las competencias y los desempeños del V ciclo para matemática en el nivel de educación primaria y todos los elementos de análisis disponibles conforme al (CNEB, 2016), vale decir el estándar de ciclo, la competencia y capacidades de área y grado.

\subsection{Ejecucion del "focus group"}

En esta se realizó con la técnica del focus group, para la propuesta de los indicadores, con los siete expertos docentes de educación primaria por un tiempo aproximado de cinco horas, siguió la secuencia.

-Introducción, presentación de los integrantes y planteamiento del objetivo.

-Formulación de las preguntas y recojo de respuestas sobre los indicadores apropiados al contexto para los componentes de la dimensión epistémica y ecológica de (EOS), que se muestran en las tablas 2 y 3 .

\section{Tabla 2.}

Preguntas para los componentes de la idoneidad epistémica. 
Componente

Situaciones-problema

Lenguajes

Reglas (Definiciones, proposiciones, procedimientos.

Argumentos

Relacionemos

Tabla 3.

Componentes de la idoneidad ecológica.

\begin{tabular}{ll}
\hline Componente & \multicolumn{1}{c}{ Preguntas para plantear indicadores } \\
\hline Adaptación al currículo & $\begin{array}{l}\text { ¿Qué acciones, productos, tareas, evidencias, preguntas y otros, } \\
\text { indicarían que el estudiante desarrolla elementos del estándar de grado } \\
\text { y la competencia "resuelve problemas de gestión de datos e } \\
\text { incertidumbre" al resolver la situación problemática contextualizada, } \\
\text { en las condiciones locales de la estrategia "aprendo en casa"? }\end{array}$
\end{tabular}

Apertura hacia la innovación didáctica.

Adaptación socio-profesional y cultural..

Educación en valores

Conexiones intra interdisciplinarias
Preguntas para plantear Indicadores

¿Qué acciones, productos, tareas, evidencias, preguntas y otros, indicarían que el estudiante usa y amplia, su conocimiento matemático al resolver la situación problemática contextualizada, en las condiciones locales de la estrategia "aprendo en casa"?

¿Qué representaciones, simbólicas, graficas, verbales, mixtas, numéricas y otras, indicarían que el estudiante aplica y amplia, su conocimiento al entender la situación problemática contextualizada, argumentando su respuesta con su propio lenguaje en las condiciones locales de la estrategia "aprendo en casa"?

¿Qué mensajes, productos, tareas, evidencias, preguntas y otros, indicarían que el estudiante entiende y aplica las definiciones y procedimientos fundamentales con claridad así como que proponga definiciones, proposiciones al resolver la situación problemática contextualizada, en las condiciones locales de la estrategia "aprendo en casa"?

¿Qué mensajes, productos, tareas, evidencias, preguntas y otros, indicarían que las explicaciones, comprobaciones son adecuadas al nivel de los estudiantes al resolver la situación problemática contextualizada, en las condiciones locales de la estrategia "aprendo en casa"?

¿Qué mensajes, productos, tareas, evidencias, preguntas y otros, indicarían que los estudiantes reconocen y utilizan los diversos significados de los objetos en sus prácticas matemáticas al resolver la situación problemática contextualizada, en las condiciones locales de la estrategia "aprendo en casa"?
Fuente: Elaboración propia en base a (Godino, 2011).
¿Qué acciones, productos, tareas, evidencias, preguntas y otros, indicarían que el estudiante tiene una práctica reflexiva, además si TICs al resolver la situación problemática contextualizada, en las condiciones locales de la estrategia "aprendo en casa"?

¿Qué acciones, productos, tareas, evidencias, preguntas y otros, indicarían que los contenidos ayudan a la formación integral del estudiante al resolver la situación problemática contextualizada, en las condiciones locales de la estrategia "aprendo en casa"?

¿Qué acciones, productos, tareas, evidencias, preguntas y otros, indicarían que los estudiantes valoran la democracia y usan el pensamiento crítico al resolver la situación problemática contextualizada, en las condiciones locales de la estrategia "aprendo en casa"?

¿Qué acciones, productos, tareas, evidencias, preguntas y otros, indicarían que los estudiantes relacionan la tarea con otros contenidos de otras áreas al resolver la situación problemática contextualizada, en las condiciones locales de la estrategia "aprendo en casa"? 
Fuente: Elaboración propia en base a (Godino, 2011).

-Escucha atenta a las intervenciones, toma de notas, sistematización preliminar.

-Conclusion y agradecimiento.

\subsection{Socializacion final de resultados}

En esta última reunión se compartió los resultados con un informe final al panel del focus group, como propuesta de indicadores de idoneidad didáctica.

\subsection{Aspectos éticos.}

Con respecto al presente estudio los aspectos éticos, ninguno de los autores tienen conflicto de intereses, por lo tanto no existe ningún riesgo de información confidencial o conflictiva.

Los docentes integrantes del focus group que participaron en la investigación, manifestaron su intención voluntaria de participar en el estudio además que lo sresultados sean expuestos a libertad de los autores, con el objetivo de compartir la información con fines educativos y divulgativos.

\section{RESULTADOS Y DISCUSIÓN}

Los profesores en el colegiado del focus group, reportaron los siguientes inidcadores de idioneidad didáctica para la evidencia de la evaluación diagnostica, en su componentes epistémico y ecológico conforme a las tablas 4 y 5.

Tabla 4.

Indicadores de idoneidad didáctica para la dimensión epistémica.

\begin{tabular}{|c|c|}
\hline Componente & Indicadores y criterios \\
\hline \multirow[t]{3}{*}{ Situaciones-problema } & Utiliza casos reales que implican mas variables (i1) \\
\hline & Relaciona las condiciones con restricciones de incertidumbre (i2) \\
\hline & Realiza inferencias y predicciones según condiciones (i3). \\
\hline \multirow[t]{3}{*}{ Lenguajes } & Traduce el lenguaje común a sistemas numéricos y algebraicos (i4). \\
\hline & Abastrae la realidad a un lenguaje matemático (i5) \\
\hline & $\begin{array}{l}\text { Adecua, unidades, expresiones, cantidades, sistemas de uso tradicional y } \\
\text { común a condiciones estadísticas validas (i6). }\end{array}$ \\
\hline \multirow{2}{*}{$\begin{array}{l}\text { Reglas (Definiciones, } \\
\text { proposiciones, } \\
\text { procedimientos. }\end{array}$} & Respeta los procedimientos según condiciones (i7). \\
\hline & $\begin{array}{l}\text { Usa deficiones conceptuales para lograr susobjetivos (i8). Adecua } \\
\text { procedimientos de calculo, representacion y deducción según condiciones (i9). }\end{array}$ \\
\hline \multirow[t]{3}{*}{ Argumentos } & Explicaciones con lenguaje cotidiano acorde al estándar de ciclo (i10) \\
\hline & $\begin{array}{l}\text { Comprobación los resultados y sugerir condiciones externas de variabilidad } \\
\text { (i11). }\end{array}$ \\
\hline & Sustenta soluciones con argumentos estadísticos (i12). \\
\hline \multirow[t]{3}{*}{ Relacionemos } & Usa términos coloquiales con términos estadísticos (i13). \\
\hline & Expresa las graficas con criterios de razonamiento en sus condiciones (i14). \\
\hline & $\begin{array}{l}\text { Utilizan las frecuencias con relación a sistema de medición de preferencias } \\
\text { (i15). }\end{array}$ \\
\hline
\end{tabular}


Fuente: Elaboración propia en base a resultados del focus group..

Tabla 5 .

Indicadores de idoneidad didáctica para la dimensión ecológica.

\begin{tabular}{ll}
\hline Componente & Indicadores y criterios \\
\hline Adaptación al currículo & Expresa la información usando medidas estadísticas y graficos (i16). \\
& Muestra procedimientos y estrategias para obtener datos y procesarlos (i17) \\
& Expone conclusiones argumentadas en información obtenida (i18). \\
Apertura hacia la & Involucrar la competencia de trabajo en entornos virtuales con las TICs (i19). \\
innovación didáctica. & Organización autónoma para alcanzar metas (i20). \\
Adaptación socio- & Sustentar comprobaciones reflexivas que argumenten las soluciones (i21). \\
profesional y cultural. & Seguir las condiciones de tarea autentica (i22). \\
& Involucrar su entorno cultural en la solución problemática (i23). \\
Educación en valores & $\begin{array}{l}\text { Intentos de complejidad y alta demanda cognitiva (i24). } \\
\text { Respeto a la cultura local y convivencia intercultural (i25). Responsabilidad } \\
\text { en las condiciones de entrega (i26). }\end{array}$ \\
& $\begin{array}{l}\text { Respeto por las ideas de los demás, responsabilidad con la información y } \\
\text { discreción con datos (i27). }\end{array}$ \\
Involucrar competencias de las áreas de Ciencia y Tecnologia (Indagacion \\
para construir conocimientos, uso de otros productos de transformación \\
interdisciplinarias \\
alimentaria) (i28). Ciencias Sociales (contexto social, gestión responsable del \\
recurso económico y del medio ambiente) (i29). \\
$\begin{array}{l}\text { Educacion para el Trabajo (relacionados a la gestión del emprendimiento } \\
\text { económico y social) (i30). }\end{array}$ \\
\hline
\end{tabular}

Fuente: Elaboración propia en base a resultados del focus group.

El conocimiento y desarrollo de la competencia resuelve problemas en gestión de datos adquiere importancia en los momentos actuales debido a que su utilidad es inmediata, por que es la responsable en la toma de desiciones que involucra el gasta de un recurso económico, material cada vez mas escaso, el uso de la estadística de modo responsable confiere a los ciudadanos el poder y la reflexión para participar con sus desiciones en la propia sociedad, los indicadores reportados en este estudio para evaluar su desarrollo en esta competencia, son favorables (Gal, 2002; Ruz et al., 2019), los docentes del focus group asumen que la competencia en estudio tiene un carácter vinculate con la convivencia de infrmacion estadística y las desiciones que se deben tomar con respecto a ellas en concordancia con (Ben-Zvi;Makar, 2016). En general los resultados muestran indicadores genéricos, son pocos los indicadores específicos que orientarían de mejor forma el trabajo para revisar una evidencia, se debe entender que asumieron el rol de proponer de acuerdo a su entendimiento del enfoque y la condición de lograr la competencia, que esta en línea con el currículo en matemática iniciando con nociones básicas y esperar inferencias estadísticas en analogía a las propuestas de (NCTM, 2000; Franklin, 2007;Franklin, 2015).

Los indicadores expuestos como resultado del estudio reflejan los intereses de los docentes del propio nivel para evidenciarlo en la prueba diagnostica de sus estudiantes, además muestran su intencionalidad de construir un instrumento adecuado y contextualizado para un uso mas versátil y dinamico (aunque no se tenga muchas particularidades, pero si generalidades), estos resultados guardan concordancia con instrumentos de esta índole reportados por (Godino et al., 2012, Godino, 2013; Godino et al., 2017), estos indicadores permitirán reorientar, adaptar, contextualizar y propiamente ejecutar procesos de análisis y estudio en la didáctica de la competencia resuelve 
problemas de gestión de datos e incertidumbre, por ende estos resultados tambien tiene concordancia con los criterios expuestos en el marco del Modelo de Cnocimientos y Competencias del profesor de matemática propuesta por (Godino et al., 2012, Godino et al., 2017).

Los resultados de indicadores de idoneidad didáctica reportados guardan relación con el logro de competencias descrito por (Tobon, 2013), porque al pretender el logro de la competencia "resuelve problemas de gestión de datos e incertidumbre" involucramos al estudiante en una complejidad de proceso para su desempeño esto es evidenciable cuando se propone los indicadores i1, i2, i3 de la dimensión epistémica e i28, i29, i30 de la dimensión ecológica. Cuando Tobon (2013) indica que la competencia es "un desempeño con idoneidad en un contexto determinado", es análogo a los indicadores propuestos en cuanto al logro eficaz, eficiente y efectivo del indicador en el contexto académico y cultural de la tarea propuesta como es la evaluación diagnostica evidenciándose con los indicadores i7,i8,i9 del componente reglas de la dimensión epistenica y los indicadores i22,i23,i24 del componente adaptación profesional y cultural de la dimensión ecológica.

Los indicadores tambien tienen analogía a los expuestos por (Arjona, 2020), los indicadores de los componentes epistémicos y ecológicos con los criterios de evaluación e indicadores para la sección de procesos, métodos y actitudes matemáticas donde para "expresa verbalmente, de forma razonada el proceso seguido en la resolución del problema" se relaciona con i4, i10, i13, i16, i21; para "utilizar procesos de razonamiento y estrategias de resolucion de problemas con cálculos y comprobación de soluciones" se relaciona con i9, i11, i17, i18, i21.

\subsection{Indicadores de idoneidad epistemica}

El logro de la competencia resuelve problemas de gestión de datos e incertidumbre, requiere que los docentes puedan asumir el rol de formadores, no solo para el desarrollo de las capacidades que aseguren el fin de la competencia, sino tambien las propias competencias matemáticas presentes en el desarrollo complejo de tareas matemáticas, estas nociones de (Rubio, 2012), son plasmadas en los indicadores del componente epistémico que no solo preveen indicadores para el logro de la competencia en si, si no que además procuran desarrollo intra y extra matemático. Los indicadores (i2,i4,i6,i8,i9,i10) incluso sugieren el conocer significaciones formales y no formales con aproximaciones intuitivas hasta entender desarrollos axiomáticos como tambien lo sugiere (Batanero y Diaz, 2007).

Al comparar los resultados de la presente investigación con el reportado por (Ruz et al.,2019) de su estudio sobre una guía de valorización para el caso de idoneidad didáctica en proceso de enseñanza-aprendizaje en didáctica de la estadística, se tiene varias coincidencias positivas aunque los indicadores propuestas tengan una generalidad es posible distinguir estas coincidencias en la componente situaciones-problema "se incluyen problemas significativos del mundo real enfatizando la variavilidad" es análogo a i1,i2,i11. Para el caso de la componente argumentos "promueve la construcción de argumentos viables, incluir conclusiones alternativas" es análogo a i11,i12. Con la componente relaciones "se promueve valorar el contexto y la variabilidad en todo el proceso, se promueve valorar los datos" es análogo a i22, i23. En el caso de reglas "inclusión de conceptos y propiedades, promover el trabajo con modelos estadisticos" es análogo con con i7,i8,i9. Para el componente lenguajes "se propone el uso de tablas y representaciones, promover el uso del lenguaje escrito" tiene analogía con i4,i5,i6. Estas analogías tienen se comparten con las nociones de competencias matemáticas como acción eficaz echa en un contexto con una finalidad expuesta por (Font, 2011).

Conforme a (Godino et al.,2017) el profesor de matemática como parte de su labor pedagógica debe analizar la experiencia matemática en la resolución problemática, de tal como que se de cuenta de las practicas, objetos y procedimientos que se da lugar en la resolución a fin de mejorar su practica para al formulación de nuevas experiencias matemáticas, y los indicadores propuestos en este estudio guardan relación ese supuesto, por que parte del análisis de los propios 
conocimientos y su aplicación en contexto. Al realizar un paralelo entre el Marco del Buen Desempeño Docente, documento del ministerio de educación del Peru con las categorías de los conocimientos didáctico.matematicos de un docente de matemática propuesto por (Godino, 2009; Pino-Fan y Godino, 2015) y los indicadores propuestos, los tres insgtrumentos tienen coincidencias sobre el manejo epistémico que debe tener el docente d ematematica para ayudar en la formación de los estudiantes e inciden en valorar sus propios procesos de estudio matemático, evidenciadas en competencias matemáticas de los docentes y que esperan de los estudiantes (Rubio, 2012).

Los indicadores propuestos deben ser entendidos desde las acciones practicas que desean los docentes del nivel de educación primaria en matemática, estos indicadores tiene semejanza con otros estudios realizados donde se plantean criterios e indicadores comunes (Godino, 2009; Godino, et al., 2017) entre otros, pero un aspecto a rescatar en la dimensión epistémica es su relación entre los que propusieron los maestros considerando que pertenecen al dominio del (CNEB, 2016) en su enfoque y perspectiva con lo análogo que resulta con otros sistemas con el sistema americano de la (NCTM, 2010) y los estándares comunes estatales de matemática (Common Core state Standards for Mathematics. CCSSM) conforme a (Scott, 2018), se presenta en la tabla 6.

\section{Tabla 6.}

Indicadores de idoneidad propuestos, estándares NCTM y practicas del CCSSM.

\begin{tabular}{lll}
\hline Indicadores propuestos & Estándares NCTM & Practicas CCSSM \\
\hline i1, i2, i3 & Resolucion de problemas & $\begin{array}{l}\text { Encontrar el sentido de problemas y } \\
\text { perseverar su solución. }\end{array}$ \\
& & $\begin{array}{l}\text { Usar herramientas apropiadas } \\
\text { estratégicamente. }\end{array}$ \\
i7 , i8, i9, i10, i11, i12 & Razonar abstracta y cuantitativamente. \\
i4, i5, i6 & Ranamiento y Demostracion & Analizar el razonamiento de otros. \\
& Comunicación & Construir argumentos vialbles \\
\hline
\end{tabular}

Fuente: elaboración propia en base a (NCTM, 2010; Scott, 2018)

De la tabla 6 podemos mencionar que se tiene valiosas coincidencias que los profesores entienden que son aspectos a trabajar desde su planificación para poder observarlas en las evidencias de los estudiantes en la evaluación.

Los indicadores de idoneidad didáctica propuestos como resultado del estudio en la presente, en su comparación con el trabajo propuesto por (Cruz et al., 2017) cual fue el planteamiento de criterios de idoneidad epistémica para el estudio de la geometría espacial en educación primaria, presentan algunas diferencias propias por el contenido temático en estudio, pero nos centraremos en las similitudes para la discusión, ambos instrumentos aportan mucha información al investigador permitiendo uma vista panorámica genérica de como se desarrolla el proceso de instrucción, logrando de esse modo la reflexión critica y la toma de decisiones em las fases de diseño, implementación y su propia evaluacion (Godino y Batanero, 1994). En el trabajo de (Cruz et al., 2017) se aprecian indicadores y critérios descritos com especificidade lo cual no ocurre en el presente estúdio, pero que en líneas genéricas tiene uma correspondência por ejemplo tanto i1,i2, i3 tienen analogia con "situaciones problemas que permitan contextualizar, ejercitar, aplicar y generalizar el conocimiento matemático" para el componente situaciones y problemas. Para Lenguajes "se favorece que los estudiantes construyan y usen sus propias representaciones para organizar, registrar y comunicar ideas" tiene analogia com i4,i5,i6 y asi en los componentes reglas, Argumentos y Relaciones, quedando establecido que los docentes em estúdio manifestaron su real intencionalidade de propuesta paralela a los reuqerimentos de uma idoneidade didactica en 
construccion desde la practica docente y con un instrumento para la educacion virtual en zonas rurales em el contexto de emergência sanitária por el Covid-19.

\subsection{Indicadores de idoneidad ecológica}

Según la revisión bibliográfica este componenete ecológico tiene una gran significatividad por las relaciones estudiantes-docentes-contexto, y desde estas relaciones pueden muy bien orientarse indicaciones para su logro efectivo ya que tienen la particularidad de orientar el currículo con factores exteriores del medio (sociales, geopolíticos, y otros) que inciden en el desarrollo de la competencia resuelve problemas de gestion de datos e incertidumbre (Godino, 2011; Rivas, 2014; Berciano, et al., 2020; Breda et al., 2020)

Para el caso de la dimensión ecológica los indicadores del presente estudio no concuerdan con el reportado por (Ruz et al.,2019) de su estudio sobre una guía de valorización para el caso de idoneidad didáctica en proceso de enseñanza-aprendizaje en didáctica de la estadística con el componente adaptación al currículo "conocen enfoques pedagógicos, normatividad de integración escolar" no tienen analogía con i16,i17,i18, esto puede ocurrir por que el focus group oriento su trabajo al logro de la competencia exclusivamente. Para el caso de la componente innovación didactica "uso de herramientas tecnológicas" con cuercan plenamente con i19,i20,i21, siendo este un indicador tambien para la competencia transversal se desenvuelve en entornos digitales (CNEB, 2016). Con la componente adaptación social y profesional "se promueve cultura de respeto, seguridad y confianza en la escuela" no tiene correlacion con i22, i23,i24, podría ser tambien por la orientación a concretar la competencia rápidamente y por la naturaleza del trabajo de (Ruz, et al., 2019). En el caso de educación en valores "promover la formación ciudadana" es completamente análogo con con i25,i26,i27, enfatizándose valores desde la actitud matemática para el ejercicio ciudadano futuro(Gal, 2002;Franklin, 2007;Ben-Zvi y Markar, 2016). Para el componente conexiones intra e interdisciplinarias "promover la integración de áreas" concuerda con i28,i29,i30, pero el indicador "promueve el trabajo colegiado entre docentes de diversas especialidades", no tiene un indicador exclusivo para este componente, dejando en evidencia que no se tiene la intencionalidad de utilizar el trabajo colegiado como un instrumento propio de construcción pedagógica para el ejercicio profesional.

El resultado de la propuesta de indicadores efectuado por los docentes en su dominio del enfoque del (CNEB, 2016) tiene relación con los estándares del sistema americano de la (NCTM, 2010) y los estándares comunes (practicos) estatales de matemática (Common Core state Standards for Mathematics. CCSSM) conforme a (Scott, 2018), se presenta en la tabla 7.

\section{Tabla 7.}

Indicadores de idoneidad propuestos, estándares NCTM y practicas del CCSSM.

\begin{tabular}{lll}
\hline Indicadores propuestos & Estándares NCTM & Practicas CCSSM \\
\hline i22, i23, i24 & Comunicación & Construir argumentos vialbles \\
i13, i14, i15, i28, i29, i30 & Conexiones & Buscar y hacer uso de estructura
\end{tabular}

Fuente: elaboración propia en base a (NCTM, 2010; Scott, 2018).

Es importante considerar en la dimensión ecológica como la relación currículo-docentesestudiantes, una construcción favorable para que se logre las competencias deseadas, por ello la analogía a nivel de los estándares de la NCTM y especialmente a nivel de las "practicas matemáticas" de los CCSSM, en la tabla 7, nos muestra una relación directa con los componentes 
de la dimensión ecológica para la adaptacio socioprofesional cultural y para las relaciones intra e intercurriculares con otras áreas, dichas practicas matemáticas adquieren significancia por que son las propias practicas docentes conscebidas como acciones ejecutables inmediatas y observables, con lo cual la actuación de los docentes del focus group tiene un nivel de calidad en su propuesta.

En el contexto actual aun de trabajo virtual remoto en las instituciones educativas en el Peru, resulta muy importante los resultados del presente estudio ya que proporciona indicadores de idoneidad didáctica justo de la dimencion ecológica que es la que relaciona el entorno con el currículo, el contexto de una educación rural con una brecha digital considerable de los lugares que tienen acceso a medios de conectividad y dominio tecnológico, resulta valioso el aporte de dichos indicadores justamente para poder contextualizar los instrumentos en este caso de la evaluación diagnostica y que estos puedan ajustarse a la sreales necesidades, intereses y condiciones de los estudiantes de educación rural, el grupo de trabajo realizo importantes aportes para que estos inidcadores sean utilizados por los docentes y que estos puedan hacer especificaciones según sus propias condiciones.

\section{CONCLUSIÓN}

El hallazo final del estudio reporta el trabajo realizado por docentes de educación primaria en su propuesta de indicadores de idoneidad didáctica para una experiencia de aprendizaje del programa "aprendo en casa" de la plataforma multicanal del ministerio de educación del Peru, que brinda el servicio de educación virtual en la emergencia sanitaria por el Covid-19, titulada "Preferencias de productos para un negocio" en el desarrollo de la competencia resuelve problemas de gestión de datos e incertidumbre de la evaluación nacional diagnostica 2021, para el quinto grado de primaria.

Proponer un sistema de indicadores de idoneidad desde el trabajo colegiado y como técnica del focus group, fue el objetivo del estudio el cual se cumplio de acuerdo a la metodología planteada teniendo un sistema de tres indicadores por cada componente, para las dimensiones epistémica y ecológica de la idoneidad didáctica.

Se propone finalmente, un sistema de indicadores de idoneidade didactica para la competência resuelve problemas de gestion de datos e incertidumbre de manera general, três por cada componente que al tener la generalidad, los maestros que hagan uso de ellas podran disponer de libertad para especificar cada uno de los indicadores conforme las condiciones de contexto em las cuales seran aplicables.

Al tener indicadores genéricos se concluye que los maestros del focus roup no tiene el conocimiento ni el domínio del enfoque ontosemiotico de la idoneidade didactica, por que se requieren especificaciones para el trabajo em aula, pudiendo incluso algunos docentes al momento de usarlos tener dificultades para especificar dichos indicadores, esta dificultad presentada es entendible ya que la cultura profesional de los maetros en las zonas rurales esta orientada al cumplimiento del Curriculo Nacional de Educacion Basica, al cual le hacen mínima critica reflexiva y analítica.

El sistema de indicadores propuestos, nunca podra ser tomada como definitiva ni trabajo conlcuido, como um aporte siempre estará sujeta a contribuciones, criticas, reflexiones , comentários que definitivamente ayudaran em su constructo, el cual si tiene el firme proposito de servir de guia a los docentes del mivel primário en el área de matemática que desde su propia practica haran um uso responsable, critico y reflexivo haciendo sus propias adecuaciones y contextualizacion segun su propia experiência em servicio.

La dificultad recurrente son los terminos técnicos y el próprio conocimiento del enfoque ontosemiotico por tener algunos aspectos teóricos abstractos que solo son posibles entenderlos com el ejercicio practico de su teoria en aula, es por ello que em esta propuesta se tiene indicadores 
genéricos. Ademas outro de los inconvenientes fue la logística virtual para el trabajo de aanalisis reflexivo y construccion de nociones, ya que hemos estado acostumbrados a um trabajo presencial para estas tareas, solo queda superarlas continuamente para la mejora de los colegiados docente.

La limitación del estudio enfocado en un tema especifico de una experiencia de aprendizaje, fue diseñada como una estrategia de construcción para la propia practica, por ello se sugiere para una siguiente oportunidad tomar el conjunto de experiencias de aprendizaje que involucre a mas competencias de modo que se puedan analizar por ejemplo la componente conexiones de la dimensión ecológico en su plenitud y desarrollo.

Se sugiere considerar un estudio con un grupo mayor de docentes y especialistas para un aporte mas constructivo y para otros instrumentos no solo de evaluación sino de propia planificación y ejecución que pueden ser trabajados en los colegiados docentes institucionales en cada institución educativa.

Agradecimiento: A los docentes participantes en el focus group, que sin su valiosa colaboración, aporte y trabajo desinteresado, no hubiese podido ser posible la realización del presente estudio.

\section{REFERENCIAS}

Arjona, P. (2020). Analisis de la idoneidad didáctica en videos educativos de Youtube sobre funciones en $2^{\circ} \mathrm{ESO}$. Tesis de fin de master. Universidad de Oviedo. TFM_PabloArjonaAndres.pdf (uniovi.es).

Arteaga, P., Batanero, C., Gea, M. (2017). La componente mediacional del conocimiento didáctico-matemático de futuros profesores sobre estadística: Un estudio de evaluación exploratorio. Educação Matemática Debate, 1(1), 54-75.

Batanero, C., Diaz, C. (2007). The meaning and understanding of mathematics. In: FRANÇOIS, K.; BENDEGEM, J. (Ed.). Philosophical Dimensions in Mathematics Education. Berlín: Ed. Springer, 107-127.

Beltrán-Pellicer, P., Godino, J. (2017). Aplicación de indicadores de idoneidad afectiva en un proceso de enseñanza de probabilidad en educación secundaria. Perspectiva Educacional, 56(2), 92-116.

Burgos, M., Godino, J.(2020). Modelo ontosemiotico de referencia de la proporcionalidad: Implicaciones para la planificación curricular en primaria y secundaria. AIEM - Avances de Investigación en Educación Matemática 2020, 18(1), 1-20.

Berciano A., Subinas A., Anasagasti J.(2020). Análisis de la idoneidad didáctica de un proyecto de estadística diseñado e implementado en un contexto en riesgo de exclusión social. AIEM Avances de Investigación en Educación Matematica-2020,18(1), 21-39.

Ben-Zvi, D., Makar, K. (2016). International Perspectives on the Teaching and Learning of Statistics. En: Ben-Zvi, D.; Makar, K. (Ed.). The Teaching and Learning of Statistics. Suiza: Springer,1-19.

Breda, A. (2020). Caracteristicas del analisi didáctico realizado por profesores para justificar la mejora en la enseñanza de las matemáticas. Bolema, Rio Claro (SP), 34(66), 69-88.

Breda, A., Font, V., Pino-Fan, L. (2018). Criterios valorativos y normativos en la didáctica de las matemáticas: el caso del constructo idoneidad didáctica. Bolema-Boletim de Educação Matemática, Rio Claro, 32(60), 255-278.

Breda, A., Lima, V. (2016) Estudio de caso sobre el análisis didáctico realizado en un trabajo final de un máster para profesores de matemáticas en servicio. REDIMAT - Journal of Research in Mathematics Education, Barcelona, 5(1),74-103.

Breda, A., Pino-Fan, L., Font, V. (2017). Meta didactic-mathematical knowledge of teachers: Criteria for the reflection and assessment on teaching practice. Eurasia Journal of Mathematics, Science \& Technology Education, 13(6), 1893-1918.

Caceres-Piñaloza, K. (2020). Educación virtual: Creando espacios afectivos, de convivencia y aprendizaje en tiempos de COVID-19. CienciAmérica, 9(2), 38-44, http://dx.doi.org/10.33210/ca.v9i2.284.

Canaza-Choque, F. (2020). Educación superior en la cuarentena global: disrupciones y transiciones. Revista Digital de Investigación en Docencia Universitaria. 14(2), e1315. https://doi.org/ 10.19083/ridu.2020.1315.

Castañeda, D.; Rodriguez, J. (2020). Covid-19-La pandemia. Una mirada a sus impactos en Latinoamerica. Revista Venezolana de Gerencia 25(91),746-753. 
Castro, M.; Paz, M., Cela, E. (2020). Aprendiendo a enseñar en tiempos de pandemia COVID-19: nuestra experiencia en una universidad pública de Argentina. Revista Digital de Investigación en Docencia Universitaria, 14(2), e1271. https://doi.org/10.19083/ridu.2020.1271

CNEB. (2016). Curriculo Nacional de Educacion Basica, Ministerio de Educacion del Peru. Lima-Peru.

Cortés, C. (2008). La técnica del Focus Group para determinar el diseño de experiencias de formación de usuarios. En Hernández Salazar, Patricia (coor.), Métodos cualitativos para estudiar a los usuarios de la información. México: UNAM, Centro Universitario de Investigaciones Bibliotecológicas.

Cruz A., Gea M., Giacomone B. (2017). Criterios de idoneidad epistémica para el estudio de la geometría espacial en educación primaria. En J. M. Contreras, P. Arteaga, G. R. Cañadas, M. M. Gea, B. Giacomone y M. M. LópezMartín (Eds.), Actas del Segundo Congreso International Virtual sobre el Enfoque Ontosemiótico del Conocimiento y la Instrucción Matemáticos. Disponible en, enfoqueontosemiotico.ugr.es/civeos.html.

D’Amore, A., Godino, J. (2007) El enfoque ontosemiótico como un desarrollo de la teoría antropológica en Didáctica de la Matemática. Revista Latinoamericana de Investigación en Matemática Educativa, 10(2), 191-218.

Diaz, G., Santos, O. (2021). El trabajo colegiado remoto y la gestión académica de dos instituciones educativas públicas, distrito de San Juan de Lurigancho - Lima, 2020. IGOBERNANZA, 4(15), $122-142$. https://doi.org/10.47865/igob.vol4.2021.130.

Díaz, J., Valdés, M. (2020). La pandemia de COVID 19 y sus implicaciones en la concepción, diseño e instrumentación didáctica de la educación médica superior cubana. Medisur [revista en Internet 18(3), 496-506. http://medisur.sld.cu/index.php/medisur/article/view/4677

Chevallard, Y. (1999). L'analyse des pratiques enseignantes en théorie anthropologique du didactique. Recherches en Didactique des Mathématiques, 19 (2), 221-266.

Font, V., Planas, N.,Godino, J. (2010). Modelo para el análisis didáctico en educación matemática. Infancia y Aprendizaje, 33(2), 89-105.

Font, V.(2011). Competencias profesionales en la formación inicial de profesores de matemáticas de secundaria. Unión, San Cristóbal de La Laguna, 26(1), 9-25.

Franke, M., Kazemi, E.,Battey, D. (2007). Mathematics teaching and classroom practice. En F.K. Lester (ed.), Second Handbook of Research on Mathematics Teaching and Learning Charlotte, NC: NCTM \& IAP.

Franklin, C. (2007). Guidelines for Assessment and Instruction in Statistics Education (GAISE) Report. 1. ed. Alexandria: American Statistical Association.

Franklin, C. (2015). Statistical Education of Teachers (SET). 1. ed. Alexandria: American Statistical Association, 2015. Disponible en: www.amstat.org/education/SET/SET.pdf.

Giacomone, B., Godino, J., Pellicer, P. (2018). Desarrollo de la competencia de análisis de la idoneidad didáctica en futuros profesores de matemática. Educ. Pesqui., São Paulo,44, e172011, http://dx.doi.org/10.1590/S16784634201844172011

Godino, J. (2013). Indicadores de la idoneidad didáctica de procesos de enseñanza y aprendizaje de las matemáticas. Cuadernos de Investigación y Formación en Educación Matemática, 11(1), 111-132.

Godino, J. (2011). Indicadores de idoneidad didáctica de procesos de enseñanza y aprendizaje de las matemáticas. XIII Conferência Interamericana de Educação Matemática (CIAEM-IACME), Recife (Brasil), 2011.

Godino, J., Batanero, C., Font, V. (2007). The ontosemiotic approach to research in mathematics education. ZDM, 39(12), 127-135

Godino, J., Batanero, C., Font, V.,Giacomone, B. (2016). Articulando conocimientos y competencias del profesor de matemáticas: el modelo CCDM. En C. Fernández, J. L. González, F. J. Ruiz, J. A. Macías, A. Jiménez, M. T. Sánchez, P. Hernández, T. Fernández y A. Berciano (Eds.), Investigación en Educación Matemática XIX (pp. 272-285). Málaga: SEIEM.

Godino, J., Bencomo, D., Font, V.,Wilhelmi, M. R. (2006) Análisis y valoración de la idoneidad didáctica de procesos de estudio de las matemáticas. Paradigma, XXVII (2), 221-252.

Godino, J., Wilhelmi, M.,Bencomo, D. (2005). Suitability criteria of a mathematical instruction process. A teaching experience of the function notion. Mediterranean Journal for Research in Mathematics Education, 4(2), 1-26.

Godino, J., Contreras, A., Font, V. (2006). Análisis de procesos de instrucción basado en el enfoque ontológicosemiótico de la cognición matemática. Recherches en Didactiques des Mathematiques, 26(1), 39-88.

Godino, J. (2002). Un enfoque ontológico y semiótico de la cognición matemática. Recherches en Didactique des Mathématiques 22, (2/3), 237-284. 
Godino, J., Font, V., Wilhelmi, M., Castro, C. (2009). Aproximación a la dimensión normativa en didáctica de las matemáticas desde un enfoque ontosemiótico. Enseñanza de las Ciencias, Barcelona, 27(1), 59-76.

Godino, J., Rivas, H., Arteaga, P. (2012). Inferencia de indicadores de idoneidad didáctica a partir de orientaciones curriculares. Práxis Educativa, 7(2), 331-354., Ponta Grossa.

Godino, J., Rivas, M., Castro, W., Konic, P. (2012). Desarrollo de competencias para el análisis didáctico del profesor de matemáticas. REVEMAT, Florianópolis, 7(2), 1-21.

Godino, J.,Giacomone, B.,Batanero, C.,Font, V.(2017). Enfoque ontosemiotico de los conocimientos y competências del professor de matemática. Revista Bolema Rio Claro 31(57), 90-113.

Gomez-Escalonilla, G., Huertas, A.,Martines, Y. (2015). Reflexiones sobre la aplicación de la técnica del focus group en el estudio de los procesos de sociabilidad juvenil en contextos multiculturales. Actas del $2^{\circ}$ Congreso Nacional sobre Metodología de la Investigación en Comunicación ISBN: 978-84-616-4124-6

Hernández, R., Fernández, C., Baptista, P. (2003) Metodología de la Investigación. Mc Graw Hill. 6ta. Edición ISBN: 978-1-4562-2396-0.

Hernández, A. (2020). Acceso, usos y problemas en la educación virtual: una aproximación a las experiencias de estudiantes y docentes durante la cuarentena obligatoria en Argetina. Pacha. Revista de Estudios Contemporaneos del Sur Global 1(1). Pp.68-75, https://doi.org/10.46652/pacha.v1i1.5.

Hummes, V., Breda, A., Seckel, M., Font, V. (2020). Criterios de idoneidad didáctica en una clase basada en el Lesson Study. Praxis \& Saber, 11(26), e-0667. https://doi.org/10.19053/22160159

Ivancovich, C.,Araya, Y. (2011). Focus Groups: tecnica de investigacion cualitativa en investigacion de mercados. Ciencias Economicas 29(1),545-554.

Jacobo, D. (2020). Reflexiones sobre la plataforma aprendo en casa del ministerio de educación del Perú durante la pandemia del Covid-19. Educación en Pandemia una visión desde la universidad, Catedra UNESCO.

Juan, S., Roussos, A. (2010). El focus gropup como tecnica de investigacion cualitativa. Serie métodos de psicología clínica $\mathrm{N}^{\circ} 09$, Universidad de Belgrano Argentina.

Kelly, A. Lesh, R., Baek, J. (Ed.) (2008). Handbook of design research in methods in education: innovations in science, technology, engineering, and mathematics learning and teaching. New York: Routledge.

Latorre, M. (2013). Diseño Curricular por capacidades y competencias en la educación superior. Universidad Marcelino Champagnat. ISBN: 978-612-4194-01-6.

Lesh, R., Sriraman, B. (2010). Re-concepttualizing mathematics education as a desing science: Sriraman, B., English, L.(Eds). Theories of Mathemathics education: seeing new frontiers. Heidelberg: Springer, 2010,123-146.

Lynch, M. (2017). What is the difference between problem, project and challenge based learning? The edvocate. http://www.theedadvocate.org/difference-problem-project-challenge-based-learning/

Malet, O.,Giacomone,B.,Repetto, A. (2021). La Idoneidad Didactica como herramienta metodológica: desarrollo y contextos de uso. Revista rememop 3(e202110),1-23, http://doir.org/10.33532/revemop.e202110.

MBDD. (2012). Marco del Buen Desempeño Docente. Ministerio de Educacion del Peru, Lima-Peru., MBDD_color1 (minedu.gob.pe).

Manzanares, A. (2008). Sobre el aprendizaje basado en problemas. En A. Escribano y Á. Del Valle (Coords.), El aprendizaje basado en problemas. Una propuesta metodológica en educación superior (pp. 17-25). Madrid: Narcea.

Meo, A.,Dabenigno, V. (2020). Teletrabajo docente durante el confinamiento por COVID-19 en Argentina. Condiciones materiales y perspectivas sobre la carga de trabajo, la responsabilidad social y la toma de decisiones. Revista de Sociología de la Educación-RASE, $14 \quad$ (1), 103-127. http://dx.doi.org/10.7203/RASE.14.1.18221.

Molina, M., Castro, E. (2011). Un acercamiento a la investigación de diseño a través de los experimentos de enseñanza. Enseñanza de las Ciencias, 29(1), 75-88.

NCTM. (2000). National Council fo Teachers of Mathematics. Principles and standards for school mathematics.

NCTM (2010). National Council of Teachers of Mathematics. The common core state standards for mathematics. Reston, VA: NCTM. Recuperado de http://www.nctm.org/uploadedFiles/Research_ News_and_Advocacy/Common_Core_Standards/CCSSM_Overview120210v.2.ppt

Nogueira, I., Fernández, T., Rodríguez, D. (2015). Análise Ontossemiótica de processos de instruçao matemática - um exemplo no Ensino Básico. Saber \& Educar, 20,174-187. 
Osorio, B. (2017). Las categorías de análisis de los conocimientos del profesor de matemáticas y los indicadores de idioneidad didáctica de los procesos de enseñanza y aprendizaje de la experiencia curricular pensamiento lógico del I ciclo de estudios de la UCV-Lima Este. Tesis de maestria Universidad Nacional de Educacion La Cantutta.

Oviedo, T., Pino, L. (2017). Competencias Didactico-Matemáticas de docentes universitarios peruanos en la enseñanza de las funciones: un estudio de caso. En VIII Congreso Iberoamericano de Educación Matemática. (pp. 152 160). JAÉN. Federación española de sociedades de profesores de Matemáticas. Recuperado de: http://www.cibem.org/images/site/LibroActasCIBEM/ComunicacionesLibroActas_CB1101-1200.pdf

Oviedo, T., Pino, L. (2017). El conocimiento didáctico-matemático en las facetas epistémica e interaccional de profesores peruanos sobre la noción de función: ejemplificando con un estudio de caso.. En Reunión Latinoamericana de Matemática Educativa. (pp. 1162 - 1170). MÉXICO. Comité Latinoamericano de Matemática Educativa A. C. . Recp. de: http://clame.org.mx/actas/

Patton, M. (2011). Developmental Evaluation: Applying Complexity Concepts to Enhance Innovation and Use. New York, NY: Guilford Press.

Perrenoud, P. (2004). Diez nuevas competencias para enseñar: Invitación al viaje. Barcelona: Graó

Pino-Fan, L., Godino, J. (2015). Perspectiva ampliada del conocimiento didáctico-matemático del profesor. Paradigma, Maracay, 36(1), 87-109.

Ramos, A., Fagúndez, Z., Castells, M. (2009). Los criterios de idoneidad y propuestas de cambios institucionales en el ámbito universitario. Investigación y Postgrado, 24(3), 115-139.

Ramos, A., Font, V. (2008). Criterios de idoneidad y valoración de cambios en el proceso de instrucción matemática. Relime, México, 11(2), 233-265.

Ravela, P., Picaroni, B., Loureiro, G. (2017) ¿Cómo mejorar la evaluación en el aula?. Reflexiones y propuestas del trabajo para docentes. Grupo Magro editores. Montevideo. ISBN:978-607-8558-39-1.

Reigeluth, C. M. (2000). ¿En qué consiste una teoría de diseño educativo y cómo se está transformando?. En C. M. Reigeluth (Ed.), Diseño de la instrucción. Teorías y modelos. Un nuevo paradigma de la teoría de la instrucción (pp. 15-40). Madrid: Santillana.

Resolucion Vice-Ministerial N¹93-2020-MINEDU. (2020). Orientaciones para la evaluación de competencias.

Rubio, N. (2012). Competencia del profesorado en el Análisis Didáctico de Prácticas, Objetos y Procesos Matemáticos.Tesis (Doctorado en Didàctica de les ciències experimentals i de la matemàtica) - Facultat de Formació del Professorat, Universitat de Barcelona, Barcelona.

Ruz, F.,Molina, E.,Contreras, J.(2019). Guia de valorización de la idoneidad didáctica de procesos de Instrucción en Didactica de la estadística. Revista Bolema 33(63), 135-154. https://doi.org/10.1590/1980-4415v33n63a07

Saavedra, B. (2019). Evaluación diagnostica. Monografia para el examen de suficiencia. Universidad Nacional de Educacion Enrique Guzman y Valle.

Sanchez, J.,Guzman, H.,Delgado, B. (2021). Produccion academica en la formación docente. Experiencia educativa durante el Covid-19. Revista de Educación Superior del Sur Global 11(1), 1-16.

Scoot, P. (2018). Reformas de los currículos escolares en Matematicas en las Americas: el caso de los Estados Unidos de America. Cuadernos de investigación y formación en educación matemática 13(17), 143-151.

Tobón.S, (2013). Integral Training and Competences. Complex thinking, curriculum, didactics and evaluation. CIFE Institute. Ecoe Editions. 4th. Bogotá edition.

Van den Heuvel-Panhuizen, M., Wijers, M. (2005) Mathematics standards and curricula in the Netherlands. Zentralblatt für Didaktik der Mathematik 37, 287-307 https://doi.org/10.1007/BF02655816.

Vera, F. (2020). La importancia del proceso de enseñanza- aprendizaje y la evaluación diagnostica, Revista Atlante Cuadernos de Educación y Desarrollo, ISSN: 1989-4155. En línea: https://www.eumed.net/rev/atlante/2020/08/evaluacion-diagnostica.html

Yepes, S., Montes, W., Álvarez, J., Ardila, J. (2017). Grupo focal: una estrategia de diagnóstico de competencias interculturales. Trilogía Ciencia Tecnología Sociedad, 10(2018), 167-181

Zavala, V., Franco, R. (2020). El Estado enseña sobre lenguas originarias: una reflexión desde el programa "Aprendo en Casa". Revista peruana de Investigación Educativa 2020, 13(1), 99-126 ISSN: 2077-4168. 